\title{
The power of a story: New, automatic associations from a single reading of a short scenario
}

\author{
FRANCESCO FORONI and ULRICH MAYR \\ University of Oregon, Eugene, Oregon
}

\begin{abstract}
The implicit association test (IAT) is typically used to assess nonconscious categorization judgments that are "under control of automatically activated evaluation" (Greenwald, McGhee, \& Schwartz, 1998, p. 1464) and that are usually considered independent of explicit judgments. The present study builds on recent work suggesting evidence of short-term modifiability of the IAT effect. Specifically, we show that reading a short text that describes a novel, fictional scenario, within which the to-be-evaluated categories are embedded, can produce substantial and immediate modulations of the IAT effect. This modulation effect does not occur when subjects are simply instructed to think about counterstereotypical associations (Experiment 1A and 1B). In Experiment 2, we use a variant of the IAT to show that scenario modulation cannot be explained in terms of strategic criterion shifts. These results suggest that a newly acquired knowledge structure targeting the abstract, category level can produce behavioral effects typically associated with automatic categorization.
\end{abstract}

A dominant theme in research on social cognition is that categorizations and evaluative judgments often occur implicitly (e.g., Bargh, 1999; Bodenhausen \& Macrae, 1998; Brewer, 1988; Devine, 1989; Fiske \& Neuberg, 1990). According to this view, people we encounter trigger automatic activation of category-specific, schematic knowledge that was acquired throughout our learning history. Such automatic categorization is not only held responsible for negative stereotypes and their behavioral consequences, but it is also deemed off-limits for explicit, verbal report. Therefore, much recent work has focused on developing behavior-based assessment techniques that allow tapping the automatic categorization system.

The most prominent method in this regard is the socalled implicit association test (IAT; Greenwald, McGhee, \& Schwartz, 1998; see also Devine, 2001), which involves intermixing two types of speeded, two-choice discriminations. The first concerns the categorization of interest. For example, participants may be asked to discriminate between names of flowers and insects. The second discrimination concerns an evaluative dimension such as that between pleasant and unpleasant words. The critical manipulation is the match between stimulus-response mappings for the two types of discriminations. For a person who favors flowers over insects, a compatible mapping implies using one key for flowers or pleasant words and the other for insects or unpleasant words. In the case of an incompatible mapping, the same key is used for

This research was funded through NIA Grant R01 AG19296-01A1, awarded to U.M. We thank Sarah Hodges and Mick Rothbart for valuable suggestions and Kim Chapman for help with data collection. Correspondence concerning this article should be addressed to U. Mayr, Department of Psychology, University of Oregon, Eugene, OR 97403 (e-mail: mayr@darkwing.uoregon.edu). flowers or unpleasant words and the other key for insects or pleasant words. The IAT effect is the response time (RT) difference between these two mapping conditions. IAT effects have been found for numerous social and nonsocial category evaluations, are usually very robust (on the order of $100 \mathrm{msec}$ or more), and are obtained even for participants who show no preferences in explicit self-report. Therefore, the IAT effect is assumed to be resistant to explicit influences and thus a relatively pure reflection of implicit, category-relevant associations (e.g., AshburnNardo, Voils, \& Monteith, 2001; Greenwald et al., 1998; McConnell \& Leibold, 2001).

The fact that much human categorization may be automatic in nature is important because automatic processing can affect thinking and action without our knowing (Blair, Ma, \& Lenton, 2001) and is usually thought to develop over time with practice. As a result, automatic categorization ought to be resistant to change (Bargh, 1999). Interestingly, however, recent work with the IAT suggests some short-term modifiability of automatic category judgments. For example, Dasgupta and Greenwald (2001) showed that after exposing participants to examples of positively viewed blacks, the typical black versus white IAT effect expressed by white subjects is reduced. Similarly, Blair et al. showed that the typically found association between females and weakness-related attributes is reduced when asking subjects to imagine a "strong woman" immediately before going through the task. According to both Dasgupta and Greenwald (2001) and Blair et al. (2001), such interventions work because they activate existing category exemplars (e.g., long-term memory records about "strong women") that otherwise are overshadowed by more prototypical members of the category. It is important to note that even evaluative judgments regarding less typical category exemplars may be 
based on an individual's learning history, possibly resulting in automatic links between a counterstereotypical exemplar and the relevant attribute (e.g., between an alien-fighting Sigourney Weaver and the attributes of strength and dominance). Thus, these results do not necessarily demonstrate direct effects of explicit representations on implicit associations (e.g., through fast formation of new associations). Rather, they demonstrate that automatic category evaluations can depend on which (automatic) associations are brought to the foreground as a function of the retrieval context.

In the present work, we tried to take the general finding that even automatic category judgments can be context dependent a step further. We assessed the effects of a very short-term intervention that was supposed to induce a knowledge structure that (1) was new rather than based on existing memory records and (2) targeted the abstract, category level rather than the specific, exemplar level. For example, in the experimental condition of Experiment 1A, participants worked through the insects/flowers IAT after reading through a half-page description of a counterstereotypical, fictional scenario. This scenario described a postapocalyptic world in which, due to radiation effects, flowers have become highly noxious, whereas insects serve as a crucial, indirect food source for humans (see the Appendix). This scenario integrates existing concepts in a new manner and in that sense induces a meaningful, though novel, conceptual network. It targets the abstract, whole-category level, rather than the specific exemplar level, because it forges a new connection between the category label and its evaluation.

Automaticity is generally thought to be a function of the amount of one's specific learning experiences (e.g., Logan, 1988). Thus, if such an intervention proves successful, it would offer a generalization of existing results (e.g., Blair et al., 2001; Dasgupta \& Greenwald, 2001) along two theoretically, particularly important dimensions: novelty (rather than based on extended practice) and abstractness (rather than based on specific exemplars) of the intervention-relevant representation.

\section{EXPERIMENT 1A}

We asked participants to work through the insects/ flowers IAT twice: once after reading the counterstereotypical scenario described in the preceding section and once after reading a prostereotypical scenario that was congruent with the stereotypical evaluations of insects and flowers as revealed with the IAT (Greenwald et al., 1998). The prostereotypical scenario was used as a control condition for the counterstereotypical scenario, describing the same situation, except that now the insects are noxious and the flowers become the critical, indirect food source.

We also wanted to make an initial attempt to examine what exactly may make a new knowledge structure an effective modulator of implicit categorizations. One possibility is that the fact that the scenarios explicitly associated the abstract category labels with either a positive or a negative value was sufficient to produce the critical ef- fect. Therefore, we included a control condition in which another group of participants received the evaluative instruction, but without the internally consistent story to support the newly established associations (see the Appendix).

\section{Method}

Participants. In this experiment, 82 students (61 females and 21 males) participated in partial fulfillment of course requirements. Of these participants, 45 were run in the scenario condition and 37 in the instruction condition.

Stimuli. The stimuli consisted of 100 words: 25 flower names, 25 insect names, 25 pleasant words, and 25 unpleasant words (from Greenwald et al., 1998).

Stimuli, Tasks, and Procedure. The participants completed the IAT as in Greenwald et al. (1998) with the only exception that they were exposed to it twice, once for each scenario in counterbalanced order. Per assessment, each participant performed a total of five blocks: (1) insects/flowers discrimination (50 trials); (2) evaluative attribute discrimination (50 trials); (3) combined, compatible discrimination (100 trials); (4) practice of reversed evaluative attribute discrimination (50 trials); and (5) combined, incompatible discrimination (100 trials). The IAT effect was obtained by comparing performance in Blocks 3 and 5, which were counterbalanced in their sequence across participants. The participants read the scenario before the first combined discrimination block (Block 3 or Block 5), and a reminder of the scenario was presented before the second combined discrimination block (Block 3 or Block 5).

Words for each block were sampled randomly without replacement from the stimulus list of 100 words ( 25 for each category). They were presented in black within a $1 \times 5$ in. gray rectangle in the center of a white computer screen. A 1,000-msec interstimulus interval (ISI) separated the end of one trial (i.e., response and disappearance of the stimulus item) and the beginning of the next trial. The participants were explicitly instructed to respond to each stimulus as fast as possible while avoiding errors.

\section{Results and Discussion}

Error trials, trials following error trials, and trials with RTs faster than $200 \mathrm{msec}$ and slower than 3,000 msec were eliminated from further analysis. RT results are presented in Figure 1 separately for the two different scenario/ instruction conditions. Apparently, there was a marked effect of scenario congruency on the IAT effect, but not of instruction congruency. The general IAT effect was highly reliable $[M=148 \mathrm{msec} ; F(1,81)=231.1, p<.001]$, as was the critical three-way interaction between mapping compatibility, scenario/instruction congruency, and scenario versus instruction $[F(1,81)=15.0, p<.001]$. In an analysis of the scenario condition alone, the scenariocongruency modulation of the IAT effect proved highly reliable $[F(1,44)=31.4, p<.001]$, but not the corresponding effect for the instruction condition $[F(1,36)=$ $.15, p>.6]$. In terms of error effects, only the IAT contrast was reliable [compatible: $M=6.7 \%$; incompatible: $M=10.9 \% ; F(1,36)=80.9, p>.001]$; the scenario manipulation produced no reliable effects.

The results of this experiment demonstrate that implicit evaluations as expressed through the IAT effect can be modulated substantially through an explicitly instructed scenario. Furthermore, consistent with the claim that novel, categorization-relevant knowledge structures can be flexibly established, we found that two opposing scenarios could be used effectively back-to-back within the same 


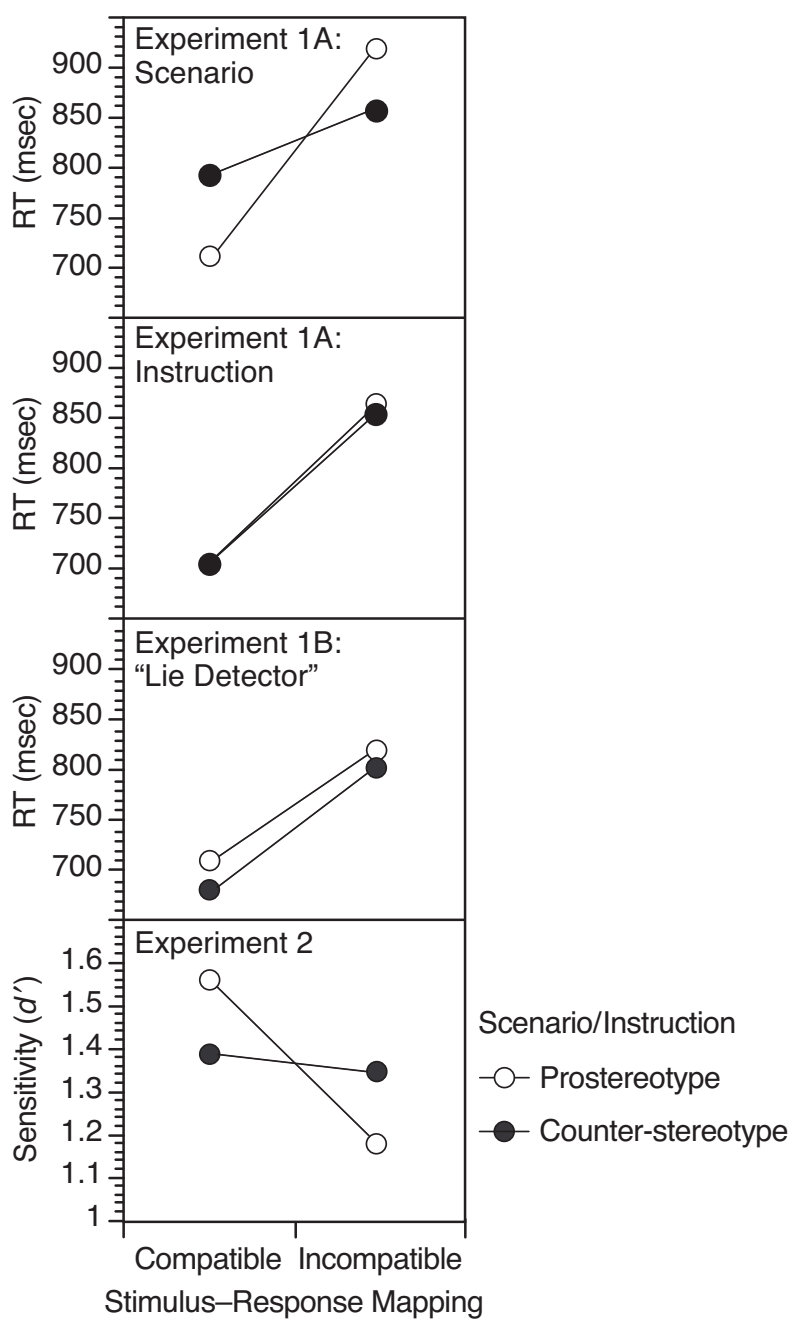

Figure 1. Response times or $d^{\prime}$ scores as a function of stimulusresponse mapping (compatible vs. incompatible with regard to the stereotypical association) and stereotype congruency of the scenario or instruction (prostereotype vs. counterstereotype) for Experiments 1A, 1B, and 2.

40-min experimental session. The results also demonstrated an important boundary condition for the IAT modulation effect. Simply instructing participants to endorse the evaluative instruction was ineffective. Thus, the IAT modulations that we did observe cannot be attributed to the component of the scenario that contains the evaluative associations per se. Rather, the effect seems to depend critically on the meaningful conceptual network within which these evaluations were embedded.

\section{EXPERIMENT 1B}

One relatively uninteresting explanation of the observed modulation is that participants may be able to somehow strategically alter the IAT effect. For example, they may produce a reduced IAT score when working with the counterstereotypical scenario by selectively slowing down responses in blocks that would be compat- ible, given the stereotype (e.g., same key for flowers and positive). Furthermore, participants may be motivated to use such a strategy through the story presented in the scenario. If this account were correct, it should be possible to compel participants to strategically "manipulate" the IAT effect by means other than through a meaningful scenario. In Experiment 1B, we introduced the IAT procedure to participants as a "lie detector" that they should try to "deceive" (see the Appendix). Thus, by explicitly stating that (1) the IAT score can be used to assess attitudes, (2) it may be possible to deceive the IAT procedure, and (3) deceiving the IAT assessment was the explicit goal, we created a situation in which participants should be at least as motivated to use strategic control as in the scenario conditions, if such control is in fact available to them.

\section{Method}

Participants. In this experiment, 32 students ( 22 females and 10 males) participated as partial fulfillment for course requirements.

Stimuli and Tasks. The same methods were used as in the instruction condition of Experiment 1A, but instruction congruency was manipulated using the lie-detector scenario (see the Appendix).

\section{Results and Discussion}

As expected, the overall pattern is almost identical to that observed in the instruction condition (see Figure 1). The basic IAT effect was highly reliable $[M=120 \mathrm{msec}$; $F(1,31)=24.1, p<.001]$. No modulation of the basic IAT pattern through the instruction-congruency factor was observed here $[F(1,31)=.63, p=.43]$. Also for errors, the general IAT effect was reliable [compatible: $M=6.3 \%$; incompatible: $M=9.3 \% ; F(1,31)=7.5, p=$ $.01]$, but no reliable instruction modulation was obtained $[F(1,31)=.02, p>.9]$.

In this experiment, we used an instruction that was supposed to compel subjects to manipulate the IAT effect through strategic criterion shifts. The fact that no IAT modulations were obtained strengthens the conclusion that the modulation obtained in Experiment $1 \mathrm{~A}$ is in fact an automatic effect of the meaningful, conceptual network induced through the scenario.

\section{EXPERIMENT 2}

Although the result of Experiment 1B speaks against strategic response slowing as a reason for the scenario effect, others have reported evidence for strategic criterion shifts playing a role in the IAT (Brendl, Markman, $\&$ Messner, 2001). Therefore, it would be useful to gain additional evidence that scenario-based modulations occur, irrespective of criterion shifts.

Nosek and Banaji (2001) recently introduced a go/no-go variant of the IAT (GNAT). The GNAT allows assessing sensitivity to certain classes of stimuli, irrespective of response criterion. Participants are asked to respond to stimuli from either of two target categories and to withhold responses to all stimuli that belong to neither category. The two target categories can either be compatibly related through a stereotypical association (e.g., flowers 
and pleasant words) or incompatibly related (e.g., flowers and unpleasant words). Sensitivity of the go/no-go response to targets has been shown to increase for compatible targets and to decrease for incompatible targets (e.g., Nosek \& Banaji, 2001). Potential scenario modulations of GNAT sensitivity effects cannot be explained as a strategic adjustment of response criteria (for a similar argument, see Blair et al., 2001). For example, if participants were to strategically slow down responding for go responses in the case of a stereotype-compatible mapping after reading the counterstereotypical scenario, this would not only lead to fewer correct go responses (consistent with a modulation effect) but also to fewer false alarms to nontargets (inconsistent with a modulation effect).

\section{Method}

Participants. Seventy-six students (54 females and 22 males) participated in the computer-based experiment in partial fulfillment of a research requirement for an introductory psychology course. The two GNAT assessments were performed following its standard procedure (see Nosek \& Banaji, 2001).

Stimuli. The stimuli consisted of 96 words: 24 flower or insect names, 24 generic words as distractors, 24 pleasant words, and 24 unpleasant words (from Greenwald et al., 1998; Nosek \& Banaji, 2001).

Procedure. The type and sequence of blocks were identical to those of Experiments $1 \mathrm{~A}$ and 1B, but instead of two-choice responses, the participants had to execute go/no-go decisions regarding the critical categories. Words were sampled randomly and without replacement from the stimulus list for each block. The participants were instructed to either press the space bar as quickly as possible for items belonging to either of the relevant categories (go trials) or withhold responding for words not belonging to the target categories (no-go trials). The responses had to occur within a $500-\mathrm{msec}$ response window. The interstimulus interval was $500 \mathrm{msec}$.

Distractors for the target category (i.e., insects or flowers) were generic words (see Nosek \& Banaji, 2001), and the distractors for the evaluative attribute were from the opposite category (e.g., pleasant words when the target was unpleasant). Each participant either worked with flowers or with insects as the critical target category. Accordingly, we used scenarios here that were constructed from those used in Experiment 1A, but we focused either on flowers or on insects without mentioning the other category (see the Appendix).

\section{Results and Discussion}

To compute the $d^{\prime}$ index for each of the combined blocks, we followed the procedure used by Nosek and Banaji (2001). Eight participants were eliminated because of below-chance performance in one or more conditions.

We obtained the basic GNAT effect, expressed in terms of $d^{\prime}$ with better performance in the compatible condition $(M=1.4)$ than in the incompatible condition $[M=$ $1.1 ; F(1,67)=28.26, p<.001$; Figure 1]. As expected, the explicitly instructed scenario affected the magnitude of the GNAT effect substantially $[F(1,67)=12.18, p=$ $.001]$. When working with the prostereotype scenario, the participants exhibited a highly reliable GNAT score $[M=.41 ; t(67)=6.71, p<.001]$, whereas when working with the counterstereotype scenario the GNAT score was not reliable $[M=.11 ; t(67)=1.58, p=.12]$.

This result replicates the scenario modulation obtained in Experiment 1A. Moreover, it demonstrates that a meaningful, conceptual network affects implicit eval- uations through changes in information-processing sensitivity rather than through strategic adaptations of response criteria.

\section{CONCLUSIONS}

We have shown here that contextual changes of automatic categorizations can be achieved by embedding the relevant categories within a conceptual network that is not only explicitly instructed, but also completely novel. Most likely, participants in our experiments have had no experience with real or fictional situations in which insects remain the sole source of nutrition and flowers become noxious. Nevertheless, effective adoption of such a new mind-set seemed to occur instantly: Immediately after participants read the half-page, fictional scenario, automatic evaluations showed strong modulations, leading a substantial portion of participants to a reversal of the usual IAT or GNAT effect in the counterstereotype condition (Experiment 1A: 28\% IAT reversals; Experiment 2: $43 \%$ GNAT reversals).

This result extends earlier findings regarding the context dependency of automatic categorization as assessed through the IAT (Blair et al., 2001; Dasgupta \& Greenwald, 2001). In these studies, the stereotypical IAT effect was reduced through interventions that activate nonstereotypical category exemplars in memory. According to our results, there is another route to the modulation of automatic categorization - namely, through embedding the abstract category label (e.g., "flowers") within a novel conceptual framework that entails a reevaluation of that category. Novelty and abstractness are theoretically important because typically, precisely the repeated exposure to specific experiences is regarded as a hallmark of automatic processing. Thus, our results provide a powerful demonstration of "conditional automaticity" (e.g., Folk, Remington, \& Johnston, 1992; Hommel, 2000; Klauer \& Musch, 2002; Neumann \& Klotz, 1994), a demonstration that raises questions about the degree to which categorization-relevant representations are truly dissociable along the explicit/controlled versus implicit/ automatic dimension.

Nevertheless, our results also show that not just any explicit mentioning of a new association has immediate, automatic effects. In fact, simply asking subjects to think of insects as positive and flowers as negative had no effect. It is therefore an important task for future work to identify the representational features that explain the qualitative jump between mere instruction of a simple association and the establishment of a meaningful conceptual network. For example, one potentially important feature of the scenarios used here is the fact that they contain a "causal theory" (e.g., radiation as the cause for flowers being noxious). Recent evidence suggests that causal relationships play a special role in defining categories (e.g., Ahn, Kim, Lassaline, \& Dennis, 2000; Medin \& Ortony, 1989), and maybe causality is a precondition for instant automaticity of category judgments. A second interesting feature of our scenarios is that they contain a strong goal- 
directed element (i.e., "distinguishing flowers from insects is critical for survival"). It is possible that active endorsement of a goal is sufficient to render goal-compatible processing automatic (e.g., Bargh, 1999; Gollwitzer, 1999). ${ }^{1}$ A final, somewhat more general aspect is that the scenario we used induced a coherent conceptual network. By definition, in coherent representations, each simple association comes packaged with other simple associations that mutually support each other (Simon \& Holyoak, 2002). For example, the simple and novel association Insects $=$ Positive is supported by the simple and novel association Insects $=$ Nutrition, and the (not explicitly stated) association Nutrition $=$ Positive. Interestingly, recent evidence suggests that many instances of complex information processing can be characterized as a process of seeking representational coherence between mutually linked concepts. According to this view, coherent representations are not only exclusive (i.e., competing coherent representations are suppressed; Mayr \& Keele, 2000) and have a self-sustaining quality (e.g., Dehaene, Kerszberg, \& Changeux, 1998), but also exert profound, implicit constraints on categorization, reasoning, and decision making (e.g., Holyoak \& Simon, 1999).

The present results do not allow a decision between these (not necessarily exclusive) possibilities. However, our finding that certain novel representations can have immediate, automatic effects suggests that the systematic examination of representational features that allow such effects is a worthwhile avenue for further research.

\section{REFERENCES}

Ahn, W.-K., Kim, N. S., Lassaline, M. E., \& Dennis, M. (2000). Causal status as a determinant of feature centrality. Cognitive Psychology, 41, 361-416.

ASHBURN-NARDO, L., VoILs, C. I., \& MONTEITH, M. J. (2001). Implicit associations as the seeds of intergroup bias: How easily do they take root? Journal of Personality \& Social Psychology, 81, 789-799.

BARGH, J. A. (1999). The cognitive monster: The case against the controllability of automatic stereotype effects. In S. Chaiken \& Y. Trope (Eds.), Dual-process theories in social psychology (pp. 361-382). New York: Guilford.

Blair, I. V., MA, J. E., \& Lenton, A. P. (2001). Imagining stereotypes away: The moderation of implicit stereotypes through mental imagery. Journal of Personality \& Social Psychology, 81, 828-841.

Bodenhausen, G. V., \& MaCrae, C. N. (1998). Stereotype activation and inhibition. In R. S. Wyer, Jr. (Ed.), Advances in social cognition: Vol. 11. Stereotype activation and inhibition (pp. 1-52). Mahwah, NJ: Erlbaum.

Brendl, C. M., Markman, A. B., \& Messner, C. (2001). How do indirect measures of evaluation work? Evaluating the inference of prejudice in the implicit association test. Journal of Personality \& Social Psychology, 81, 760-773.

BREWER, M. B. (1988). A dual process model of impression formation. In T. K. Srull \& R. S. Wyer, Jr. (Eds.), Advances in social cognition: Vol. 1. A dual process model of impression formation (pp. 1-36). Hillsdale, NJ: Erlbaum.

Dasgupta, N., \& Greenwald, A. G. (2001). On the malleability of automatic attitudes: Combating automatic prejudice with images of ad- mired and disliked individuals. Journal of Personality \& Social Psychology, 81, 800-814.

Dehaene, S., Kerszberg, M., \& Changeux, J.-P. (1998). A neuronal model of a global workspace in effortful cognitive tasks. Proceedings of the National Academy of Sciences, 95, 14529-14534.

DEvine, P. G. (1989). Stereotypes and prejudice: Their automatic and controlled components. Journal of Personality \& Social Psychology, 56, 5-18.

DEVINE, P. G. (2001). Implicit prejudice and stereotyping: How automatic are they? Journal of Personality \& Social Psychology, 81, $757-$ 759.

Fiske, S. T., \& NeuberG, S. L. (1990). A continuum of impression formation, from category-based to individuating processes: Influences of information and motivation on attention and interpretation. In M. P. Zanna (Ed.), Advances in experimental social psychology (Vol. 23, pp. 1-74). San Diego: Academic Press.

FolK, C. L., Remington, R. W., \& Johnston, J. C. (1992). Involuntary covert orienting is contingent on attentional control settings. Journal of Experimental Psychology: Human Perception \& Performance, $\mathbf{1 8}$, 1030-1044.

Gollwitzer, P. M. (1999). Implementation intentions: Strong effects of simple plans. American Psychologist, 54, 493-503.

Greenwald, A. G., McGhee, D. E., \& Schwartz, J. L. K. (1998). Measuring individual differences in implicit cognition: The implicit association test. Journal of Personality \& Social Psychology, $\mathbf{7 4}_{2}$ 1464-1480

HoLYOAK, K. J., \& SimON, D. (1999). Bidirectional reasoning in decision making by constraint satisfaction. Journal of Experimental Psychology: General, 128, 3-31.

Hommel, B. (2000). The prepared reflex: Automaticity and control in stimulus-response translation. In S. Monsell \& J. Driver (Eds.), Control of cognitive processes: Attention and performance XVIII (pp. 247273). Cambridge, MA: MIT Press.

Klauer, K. C., \& Musch, J. (2002). Goal-dependent and goal-independent effects of irrelevant evaluations. Personality \& Social Psychology Bulletin, 28, 802-814.

Logan, G. D. (1988). Toward an instance theory of automatization. Psychological Review, 95, 492-527.

MAYR, U., \& KeELE, S. (2000). Changing internal constraints on action: The role of backward inhibition. Journal of Experimental Psychology: General, 129, 4-26.

MCConNell, A. R., \& LEIBOLD, J. M. (2001). Relations among the implicit association test, discriminatory behavior, and explicit measures of racial attitudes. Journal of Experimental Social Psychology, 37, 435-442.

MEDIN, D. L., \& Ortony, A. (1989). Psychological essentialism. In S. Vosniadou \& A. Ortony (Eds.), Similarity and analogical reasoning (pp. 179-195). New York: Cambridge University Press.

Neumann, O., \& Klotz, W. (1994). Motor responses to nonreportable, masked stimuli: Where is the limit of direct parameter specification? In C. Umiltà \& M. Moscovitch (Eds.), Attention and performance $X V$ : Conscious and nonconscious information processing (pp. 123150). San Diego: Academic Press.

Nosek, B. A., \& BanaJi, M. R. (2001). The go/no-go association task. Social Cognition, 19, 625-666.

SIMON, D., \& HOLYOAK, K. J. (2002). Structural dynamics of cognition: From consistency theories to constraint satisfaction. Personality \& Social Psychology Review, 6, 283-294.

\section{NOTE}

1. The absence of a modulation effect for the lie-detection intervention (Experiment 1B), which uses an explicit goal (namely, to try to deceive the lie detector), provides some suggestion that a goal per se does not necessarily determine implicit categorization. 


\section{APPENDIX}

\section{Experiment 1A: Counterstereotype Scenario}

Imagine you are a survivor of a nuclear war. Years after the war, the radiation still affects your life. The only food resources you have are certain higher-level animals (e.g., sheep, cows). You can breed them but you have to make sure that they don't eat or touch any flowers. All flowers are radioactive and they cannot be used as nutrition for your animals. The only way you have to raise your animals is to feed them with all kinds of insects. In fact, because of their fast metabolism and genetic mutations, insects have already overcome the radiation problems. In this desolate scenario your survival completely depends on the insects. They are the most positive things you can imagine. In contrast, flowers are poison for you and your animals. They are the most negative things you can imagine. To survive under such circumstances, it is extremely important to make quick and accurate choices regarding flowers versus insects. Imagine the following is a tutoring program that is supposed to train people to make these kinds of choices. Its goal is to establish firmly in people's minds, even in difficult and misleading situations: Flowers $=$ Negative, Insects $=$ Positive. Thus, while performing the task, please try to do so with this scenario in mind.

\section{Experiment 1A: Counterstereotype Instruction}

Imagine the following is a tutoring program that is supposed to train people to make quick and accurate choices. Its goal is to establish firmly in people's minds, even in difficult and misleading situations: Flowers $=$ Negative, Insects $=$ Positive. Thus, while performing the task, please try to do so with this association in mind.

\section{Experiment 1B: Counterstereotype "Lie-Detector" Instruction}

Imagine the following is a "lie-detector program" and your behavior in the next block of trials can be used as a kind of "lie detector." Your goal is to try to deceive the lie detector. For the next block of trials, imagine you need to pretend that you are a person who likes insects very much and strongly dislikes flowers. Perform the following task (as fast and accurately as possible) while trying everything you can to act like a person who believes: Insects $=$ Positive, Flowers $=$ Negative.

\section{Experiment 2: Counterstereotype Scenario for Flowers as Target Category}

Imagine you are a survivor of a nuclear war. Years after the war, the radiation still affects your life. The only food resources you have are certain higher-level animals (e.g., sheep and cows). You can breed them but you have to make sure that they don't eat or touch any flowers. All flowers are radioactive and they cannot be used as nutrition for your animals. In this desolate scenario, flowers are poison for you and your animals. They are the most negative things you can imagine. To survive under such circumstances, it is extremely important to make quick and accurate choices regarding flowers. Imagine the following is a tutoring program that is supposed to train people to make these kinds of choices. Its goal is to establish firmly in people's minds, even in difficult and misleading situations: Flowers $=$ Negative. Thus, while performing the task, please try to do so with this scenario in mind.

Note-All prostereotype texts were created by inverting the roles of the critical categories (e.g., flowers take the role of insects and insects the role of flowers). The Experiment 2 scenarios used only one target category and were created by deleting portions of the scenario used in Experiment $1 \mathrm{~A}$ that referred to the category that was not the target. 